\title{
Caracterización ambiental, demografía y amenazas para su conservacion de la población lucense de Culcita macrocarpa C. Presl. (NW ibérico)
}

Recibido: 25 xaneiro 2011 / Aceptado: 5 setembro 2011

(C) IBADER- Universidade de Santiago de Compostela 2011

\begin{abstract}
Resumen Se aportan datos sobre el biotopo y parámetros demográficos de la única población conocida hasta este momento de Culcita macrocarpa C. Presl. en la Provincia de Lugo (Galicia, NW España). Los individuos se distribuyen en tres subpoblaciones de tamaño desigual (19, 21 y 1 plantas) que crecen entre los 460 y 500 m en ambientes sombríos al lado de un curso de auga temporal que discurre por el interior de un extenso robledal. En conjunto, la proporción de individuos juveniles y adultos es muy semejante, mostrando los ejemplares una elevada vitalidad, aunque algo más del $10 \%$ de sus frondes están afectadas por la pérdida de superficie fotosintética de origen desconocido. Actualmente no existen factores de amenaza evidentes, aunque la sustitución del bosque en el que se encuentra la población estudiada por plantaciones de crecimiento rápido - la intensificación del pastoreo podrían afectar negativamente su conservación. Por esta razón, se propone la declaración de un Espacio Natural Protegido en la cuenca del Río Sor para asegurar la supervivencia de esta población de un helecho protegido por su condición de especie amenazada de extinción.
\end{abstract}

Palabras clave caracterización ecológica, endemismo ibero-macaronésico, demografía, especie amenazada, NW Península Ibérica.

Manuel A. Rodríguez Guitián · G. Lijó Pose

Departamento de Produción Vexetal. Escola Politécnica Superior. USC. Campus Universitario s/n. 27002-Lugo.

E-mail: manuelantonio.rodriguez@usc.es

Javier Ferreiro da Costa

Instituto de Biodiversidade Agraria e Desenvovemento Rural.

IBADER. Campus Universitario s/n. 27002-Lugo

Pablo Ramil-Rego

Departamento de Botánica. Escola Politécnica Superior. USC.

Campus Universitario s/n. 27002-Lugo
Abstract Data on biotope characteristics and demography of the unique Culcita macrocarpa C. Presl. population known in Lugo province (Galice, NW Spain) are presented. The plants form three sub-populations with 19, 21 and 1 individuals between 450 and $500 \mathrm{~m}$ a.s.l. and grow in a shady and wet biotope near a temporay stream that runs into a wide common oak-dominated forest. Proportion of adult and juvenile plants are similar and they show great vitality in general. Nevertheless, almost $10 \%$ of the fronds show a more or less severe loss of photosynthetic tissue caused by an unkown agent. Nowadays there is no outstanding threats that menace this population, but replacement of native woodlands by rapid-growth species plantations or increase cattle preassure could affect in a negative way its future preservation. We suggest that the better action to ensure the preservation of the studied population of this endangered fern is to included the site into a protected area for conservation.

Key words ecological characterisation, ibero-macaronesian endemism, demography, threatened fern, NW Iberian Peninsula.

\section{Introducción y objetivos}

Culcita macrocarpa C. Presl es un pteridofito endémico de distribución ibero-macaronésica cuya supervivencia se encuentra amenazada en la mayor parte de su área de distribución, siendo considerada dentro del territorio español como "en peligro de extinción" (EN) en el "Atlas y Libro Rojo de la Flora Vascular Amenazada de España" (Bañares et al. 2004). Desde el punto de vista legal, a nivel comunitario figura dentro de los anexos II (las especies animales y vegetales de interés comunitario para cuya conservación es necesario designar zonas especiales de conservación) y IV (especies animales y vegetales de interés comunitario que requieren una protección estricta) de la DC 92/43/CEE, mientras que las poblaciones gallegas se encuentran legalmente protegidas en virtud de su inclusión, dentro de la categoría de "En peligro de extinción", en el Catálogo Galego de Especies Ameazadas (Xunta 2007). 
Aunque su existencia en territorio gallego es conocida desde mediados del pasado siglo (Laínz 1968), la presencia de esta especie en la provincia de Lugo (Muras, Ambosores, A Fraga Gorda, 29TPJ0220; figura 1a) ha sido dada a conocer en época reciente por Romero et al. (2004), constituyendo la localidad más oriental de las conocidas hasta la fecha en el $\mathrm{N}$ de Galicia, que se une a las previas de Serra da Capelada, Serra de Forgoselo y cuencas bajas de los ríos Eume y Mera.

La situación relativamente alejada de este núcleo poblacional con relación a las localidades más próximas coruñesas y la escasez de información acerca sus condiciones ambientales y aspectos demográficos nos ha motivado a realizar una caracterización preliminar del medio en que se desarrolla, un recuento y estudio biométrico de sus efectivos poblacionales así como una valoración preliminar de los factores que pudieran poner en peligro su supervivencia. Este tipo de información resulta imprescindible para llevar a cabo los trabajos de monitorización y seguimiento a largo plazo de las poblaciones de este helecho protegido a que obliga el Artículo 17 la DC 92/43/CEE, así como la elaboración del Plan de Recuperación que establece la "Lei 9/2001 de Conservación da Natureza de Galicia" para todas las especies catalogadas como EN ("en peligro de extinción") dentro del ámbito territorial gallego.

\section{Metodología}

El presente trabajo se ha planteado en dos fases: una primera de recogida de datos, relacionados con la caracterización ambiental y fitocenótica, el conteo de individuos y la toma de datos biométricos, y otra posterior, de análisis de la información. En la medida de lo posible, para la caracterización biológica y reproductiva de las (sub)poblaciones estudiadas se han seguido los criterios aplicados en el "Atlas y Libro Rojo de la Flora Vascular Amenazada de España" (Bañares et al. 2004), si bien se han introducido algunas modificaciones con la finalidad de analizar adecuadamente la información recopilada. En particular, se ha considerado como un único individuo a cada conjunto de caules que se mantienen interconectados en el momento de la toma de datos. En cada uno de ellos se ha determinado el biotopo en el que se encuentra (diferenciando entre medio rupícola [R], pie de cantil rocoso [P] y borde de cauce fluvial [C])(figura 1b), su condición reproductiva (estado vital vegetativo o fértil), número y longitud de frondes y estado fitosanitario. Igualmente, se han determinado los posibles factores de amenaza que pudieran incidir negativamente en la supervivencia de dichas poblaciones. Los trabajos de campo se realizaron en el primer semestre del año 2005.

\section{Resultados y discusión}

La información de carácter ambiental, biométrica y demográfica recopilada en el campo se muestra en el Anexo que figura al final del trabajo. A partir de ella se han elaborado diversas tablas y figuras por medio de las cuales se facilita la interpretación de dichos datos.

\section{Situación fisiográfica y caracterización ambiental}

Aunque en la cita de Romero et al. (2004) se especificaba una altitud de $480 \mathrm{~m}$ para la localidad lucense de Culcita macrocarpa, el estudio detallado de la situación de los individuos existentes muestra que en realidad se trata de tres subpoblaciones (FG-I, FG-II y FG-III) separadas unos $150 \mathrm{~m}$ en línea recta entre sí y distribuídas altitudinalmente entre los 460 y los $500 \mathrm{~m}$ de altitud (tabla 1). Dichas subpoblaciones se encuentran dentro de una masa arbolada de unas 45 ha de superficie, conocida localmente como A Fraga Gorda, que se extiende a lo largo de una pequeña cuenca fluvial por la que discurre un pequeño curso estacional, situada en la cabecera del Río Sor (figuras 1,2 y 3 ). El rango altitudinal comentado constituye el techo altimétrico para esta especie en Galicia, encontrándose unos $200 \mathrm{~m}$ por encima de las poblaciones conocidas a mayor altitud de las cuencas de los ríos Eume, Xubia, Landoi y Mera, en la vecina provincia de A Coruña. No obstante, hacia el centro de la Cornisa Cantábrica este nivel se ve superado en algunas localidades asturianas situadas en la cuenca del Río Deva, en las que este helecho alcanza los 650 m de altitud (Argüelles et al. 2004-2005)
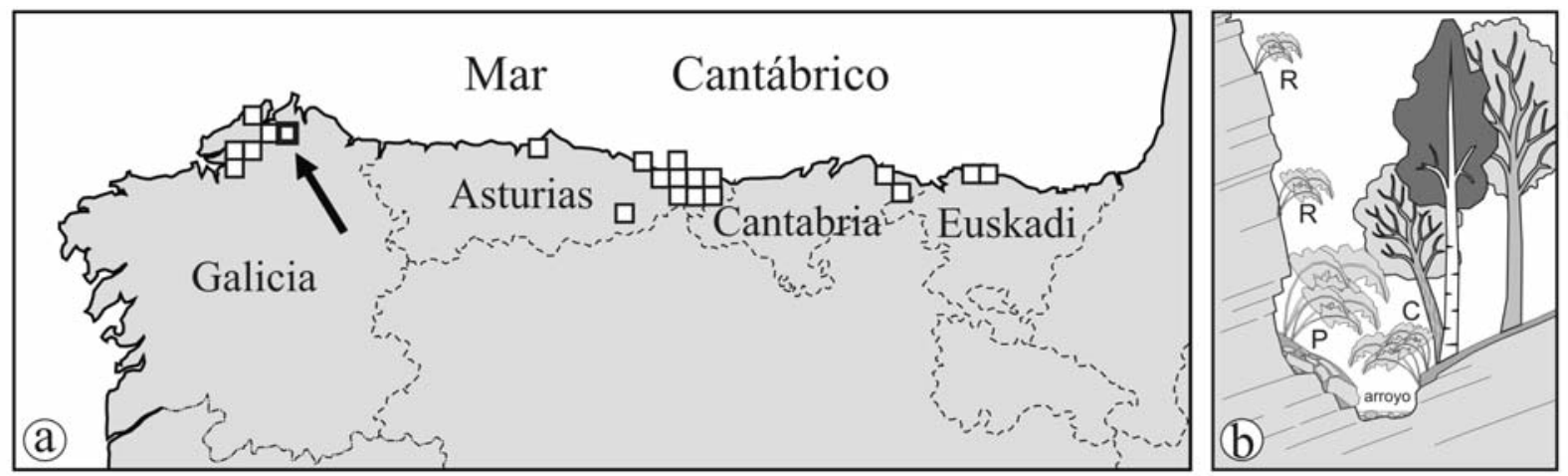

Figura 1.- a: Localización de la población de Culcita macrocarpa C. Presl. estudiada (cuadrado señalado por la flecha) en el contexto cántabro-atlántico septetrional ibérico. b: posiciones topográficas en las que crecen los individuos de Culcita macrocarpa en la población estudiada. R: pared rocosa; P: pié de cantíl; C: márgen de arroyo 
Según la caracterización bioclimática de Galicia propuesta por Rodríguez Guitián \& Ramil Rego (2007), la población del valle del Río Sor se localizaría dentro del termotipo mesotemplado inferior, correspondiéndole un ombrotipo húmedo superior y un bioclima subhiperoceánico, fuertemente influenciado durante la época estival por la condensación de masas nubosas procedentes del Mar Cantábrico. Tomando como referencia los valores estimados por Martínez Cortizas et al. (1999) para las estaciones meteorológicas de Grañas do Sor (Mañón, A Coruña) y Xan Branco (Ourol, Lugo), ambas prácticamente equidistantes en línea recta unos $7 \mathrm{~km}$ de las poblaciones estudiadas y situadas a 453 y $520 \mathrm{~m}$ de altitud respectivamente, puede establecerse un promedio de 1500$1600 \mathrm{~mm}$ anuales de precipitación para el área montañosa en la que se encuentra el núcleo poblacional de Culcita macrocarpa estudiado, de los que aproximadamente 130150 corresponderían a los meses estivales (junio-agosto).

Como se muestra en la tabla 1 y en las figuras 2 y 3 , estas poblaciones se desarrollan en un microambiente relativamente variado. Tanto en la subpoblación inferior (FG-I) como en la intermedia (FG-II), se encuentran individuos en posición de pié de cantil (biotopo $\mathrm{P}$ ) y otros

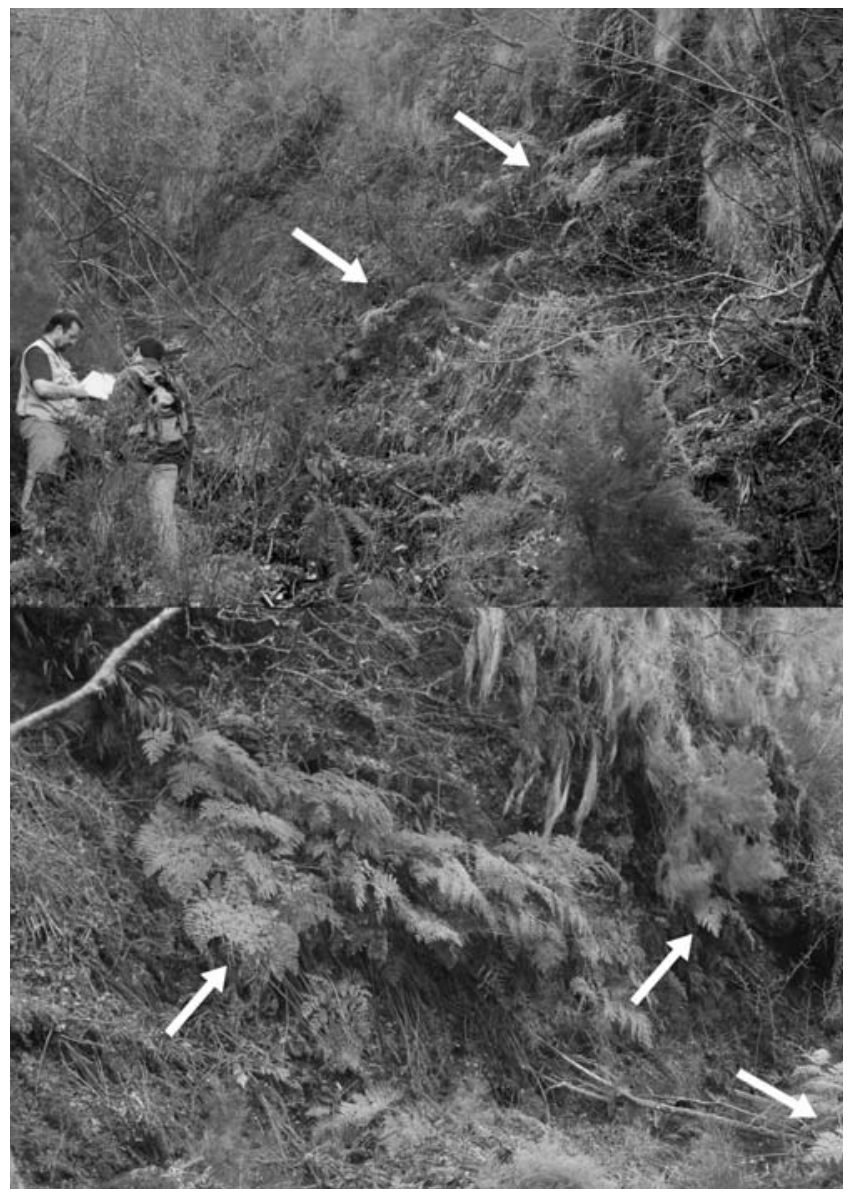

Figura 2.- Aspecto de las subpoblaciones de Culcita macrocarpa estudiadas. Arriba: FG-I; abajo FG-II. Las flechas indican algunos de los individuos integrantes de cada población enraizados directamente en la pared rocosa adyacente (biotopo R), en ambos casos de naturaleza pizarrosoesquistosa (IGME 1976), mientras que la subpoblación superior (FG-III) está representada por un único individuo ramificado de gran tamaño situado en un pequeño talud terroso que conforma la margen derecha del pequeño arroyo que circula por el valle. Los individuos asentadas al pie de los cantiles o en el margen de arroyo enraízan en un horizonte edáfico húmedo y con abundantes restos de materia orgánica, en gran medida generados por la descomposición de frondes viejas. En el caso de los asentados en las paredes rocosas, los rizomas crecen aferrándose a las pequeñas repisas y grietas que presenta el sustrato.

Culcita macrocarpa es un helecho de carácter higrófilo y umbrófilo cuyas poblaciones septentrionales ibéricas tienden a ser más numerosas y con mayor vitalidad bajo una cubierta arbórea densa y en las inmediaciones de pequeñas cascadas o tramos fluviales rápidos, en los que se mantenga un elevado grado de humedad atmosférica (Boudrie 1998, Prelli 2002, Quintanilla et al. 2004). De todas maneras, también puede crecer en formaciones vegetales que carecen de cubierta arbórea, siempre que se trate de

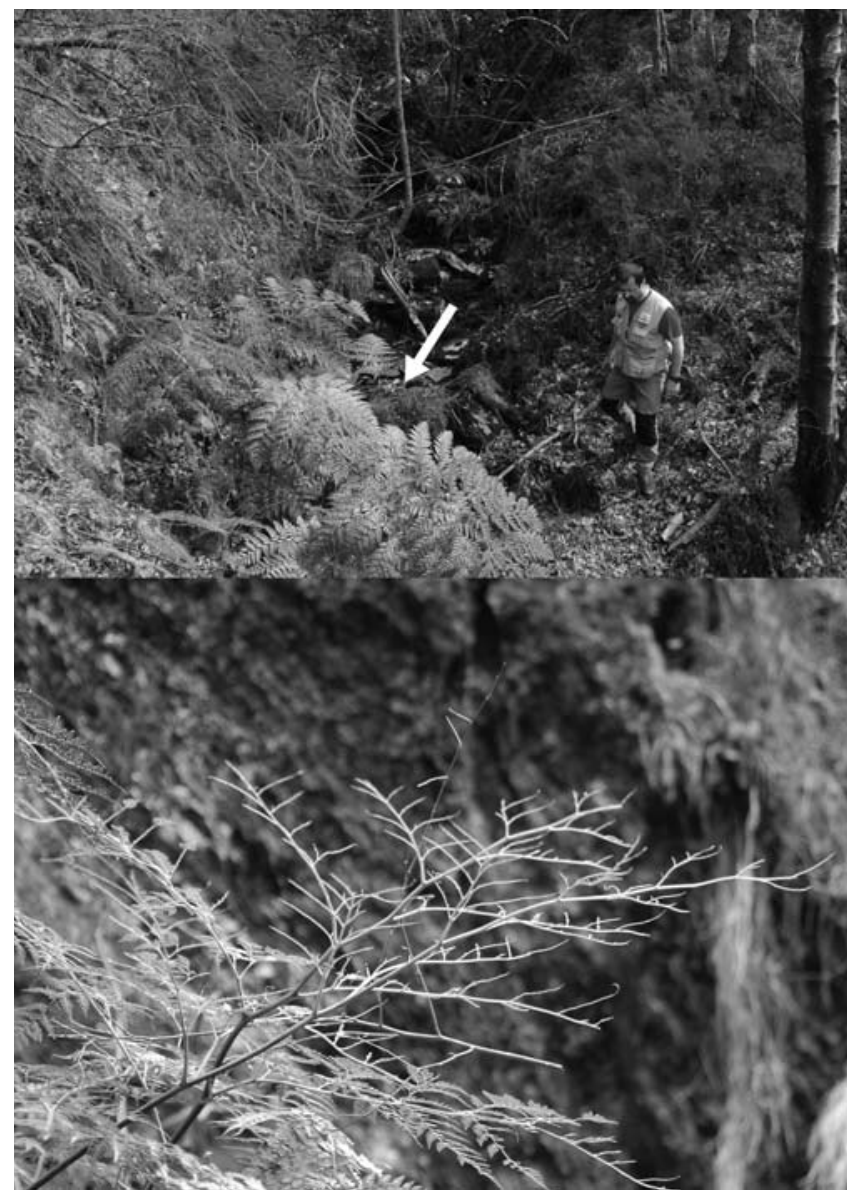

Figura 3.- Aspecto de la subpoblación FG-III (arriba) y de una fronde defoliada por un agente desconocido (abajo) 


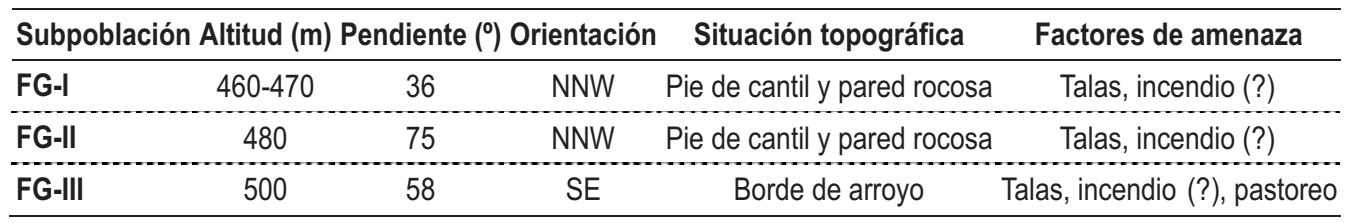

Tabla 1.- Caracterización ambiental y factores potenciales de amenaza identificados en cada subpoblación de Culcita macrocarpa estudiada

enclaves con una atmósfera saturada en vapor de agua, como torrenteras (Pérez Carro et al. 1989). En el caso de las tres subpoblaciones estudiadas, la protección frente a la desecación creemos que está más vinculada a la sombra proporcionada por las paredes rocosas o la propia masa arbolada en la que se encuentran inmersas que el hecho de situarse en las proximidades de un pequeño cauce fluvial estacional. La influencia microclimática favorable para la especie derivada del efecto regulador que ejerce el dosel de los bosques mesófilos que la albergan, especialmente durante la época estival, ha sido discutida en profundidad por Amigo (2005) a partir de la monitorización ambiental de varias poblaciones de Culcita macrocarpa presentes en localidades del Parque Natural de las Fragas do Eume (A Capela-Monfero, A Coruña) y la cabecera del Río Castro (San Sadurniño, A Coruña).

\section{Caracterización fitocenótica}

Las poblaciones estudiadas de Culcita macrocarpa se integran en comunidades relativamente pobres en plantas vasculares dominadas por pteridofitos, como Athyrium filixfemina, Blechnum spicant, Dryopteris dilatata, $D$. affinis, $D$. aemula u Osmunda regalis (tabla 2). El resto de taxones presentes (Avenella flexuosa, Brachypodium sylvaticum, Erica arborea, Erica mackaiana, Frangula alnus, Hedera hibernica, Holcus mollis, Lonicera periclymenum, Oxalis acetosella, Rubus sp., Saxifraga lepismigena, S. spathularis, Vaccinium myrtillus), de ocurrencia más irregular y cobertura sensiblemente menor, son principalmente plantas vasculares comunes en comunidades de matorral y bosques del entorno. Como puede apreciarse en la citada tabla, la flora vascular que acompaña al pteridofito estudiado en las tres subpoblaciones analizadas se asemeja bastante a la establecida por Fernández Ordoñez et al. (2003) para la "comunidad de Culcita macrocarpa" del oriente de Asturias.

En general, las poblaciones de Culcita macrocarpa de la Cornisa Cantábrica se encuentran en ambientes nemorales abrigados, húmedos y umbrosos (Viane et al. 1987, Amigo \& Norman 1993, 1995, Boudrie 1998, Quintanilla \& Amigo 2001, Quintanilla et al. 2002, Fernández Ordoñez et al. 2003). Para el caso de Galicia son reiteradas las referencias a avellanedas y robledales de vaguada o "robledales mixtos", identificados por diferentes autores con la subasociación pulmonarietosum longifoliae de los bosques mayoritarios en el territorio (Blechno spicant-Quercetum
roboris)(Laínz 1968, Amigo \& Norman 1993, 1995, Quintanilla 1997), comunidad cuya significación fitosociológica ha sido reinterpretada recientemente como variante por Rodríguez Guitián (2010a). Más recientemente, se ha documentado la presencia de individuos aislados o pequeñas poblaciones de esta especie en el sotobosque de bosques mixtos acidófilos (asociación Polysticho setiferiAceretum pseudoplatani inéd.)(Rodríguez Guitián 2004, 2005) y avellanedas-saucedas y fresnedas riparias (asociaciones Hyperico androsaemi-Coryletum avellanae y Valeriano pyrenaicae-Fraxinetum excelsioris)(Rodríguez Guitián (2010b). Más hacia el E, desde el oriente de Asturias hasta el País Vasco, las comunidades con Culcita macrocarpa aparecen principalmente en ambientes de alisedas riparias silicícolas (Hyperico androsemi-Alnetum glutinosae subas. osmundetosum regalidis)(Fernández Ordoñez et al. 1984, Pérez Carro et al. 1989), habiéndose citado su presencia en bosques mixtos riparios sobre sustratos ácidos en un trabajo más reciente (Fernández Ordoñez et al. 2003).

Como ya se ha señalado con anterioridad, la particular situación en la que se encuentran las dos primeras subpoblaciones del helecho estudiadas, al abrigo de sendas paredes rocosas y sin dosel arbóreo directo que las proteja, dificulta su interpretación desde el punto de vista fitocenótico como un aspecto particular de una comunidad arbórea. En este sentido, si se tiene en cuenta el claro predominio en la comunidad de helechos, su carácter higroesciófilo y su desarrollo sobre roquedos y suelos húmicos poco desarrollados (comunidades exocomófitas), podría considerarse que se trata de una comunidad perteneciente a la alianza Hymenophyllion tunbrigensis (orden Anomodonto-Polypodietalia, clase AnomodontoPolypodietea), tal y como sugieren Fernández Ordoñez et al. (2003) para Asturias. En el caso de la tercera subpoblación, sí existe un dosel continuo por encima del biotopo ocupado por esta especie que, sobre el talud terroso del margen del arroyo en el que se encuentra, está constituído por pies dispersos de Betula pubescens, Corylus avellana, Ilex aquifolium, Sorbus aucuparia y Frangula alnus. Aunque no poseemos un inventario florístico detallado del conjunto de la comunidad arbolada en la que inserta esta tercera subpoblación, en principio podría asimilarse a los abedulares de cabecera fluvial del $\mathrm{N}$ de Galicia y NW de Asturias (asociación Violo palustrisBetuletum pubescentis), recientemente descritos por Rodríguez Guitián (2010b). 


\begin{tabular}{lcccc}
\hline Población & FG-I & FG-II & FG-III & SINT. \\
Area de inventario $\left(\mathbf{m}^{2}\right)$ & 20 & 25 & 8 & $2-25$ \\
Cobertura de la vegetación (\%) & 100 & 100 & 100 & $90-99$ \\
Altura de la vegetación $(\mathbf{m})$ & 2,0 & 1,8 & 1,0 & --- \\
No de taxones (sólo plantas vasculares) & $\mathbf{1 4}$ & $\mathbf{7}$ & $\mathbf{1 3}$ & $\mathbf{1 0}$ \\
Columna & $\mathbf{1}$ & $\mathbf{2}$ & $\mathbf{3}$ & $\mathbf{4}$ \\
\hline Dosel arbóreo & & & & \\
\hline Frangula alnus & 2 & $\cdot$ & $\mathrm{P}$ & 2 \\
Betula pubescens & $\cdot$ &. & $\mathrm{P}(\mathrm{dom})$ & 3 \\
Corylus avellana & $\cdot$ &. & $\mathrm{P}$ & 2 \\
llex aquifolium & $\cdot$ &. & $\mathrm{P}$ & 1 \\
Quercus robur & $\cdot$ &. & $\cdot$ & 1 \\
Castanea sativa & $\cdot$ &. &. & 1 \\
Sorbus aucuparia & $\cdot$ &. & $\mathrm{P}$ &. \\
\hline
\end{tabular}

\begin{tabular}{lllll}
\hline Otras especies & & & & \\
\hline Culcita macrocarpa & 3 & 5 & 3 & 4 \\
Blechnum spicant & 2 & 1 & 2 & 4 \\
Dryopteris dilatata & 2 & 1 & 1 & 2 \\
Lonicera periclymenun & 1 & + & 1 & 1 \\
Rubus sp. & + & + & + & $\cdot$ \\
Dryopteris affinis & 3 & $\cdot$ & 1 & 3 \\
Vaccinium myrtillus & + & $\cdot$ & + & 2 \\
Athyrium filix-femina & 1 & 1 & $\cdot$ & $\cdot$ \\
Dryopteris aemula & 1 & $\cdot$ & 1 & $\cdot$ \\
Oxalis acetosella & 1 & $\cdot$ & $\cdot$ & 2 \\
Hedera hibernica/helix & $\cdot$ & $\cdot$ & 1 & $3^{*}$ \\
Erica arborea & $\cdot$ & $\cdot$ & + & 3 \\
Holcus mollis & 1 & $\cdot$ & $\cdot$ & $\cdot$ \\
Osmunda regalis & 1 & $\cdot$ & $\cdot$ & $\cdot$ \\
Saxifraga spathularis & 1 & $\cdot$ & $\cdot$ & $\cdot$ \\
Saxifraga lepismigena & $\cdot$ & + & $\cdot$ & $\cdot$ \\
Avenella flexuosa & $\cdot$ & $\cdot$ & 1 & $\cdot$ \\
Brachypodium sylvaticum & $\cdot$ & $\cdot$ & + & $\cdot$ \\
Erica mackaiana & $\cdot$ & $\cdot$ & + & $\cdot$ \\
Daboecia cantabrica & $\cdot$ & $\cdot$ & $\cdot$ & 2 \\
Luzula sylvatica & $\cdot$ & $\cdot$ & $\cdot$ & 2 \\
Cystopteris fragilis & $\cdot$ & $\cdot$ & $\cdot$ & 1 \\
Woodwardia radicans & $\cdot$ & $\cdot$ & $\cdot$ & 1 \\
\hline
\end{tabular}

Tabla 2.- Comparación de la composición florística de la vegetación correspondiente a cada una de las subpoblaciones de Culcita macrocarpa estudiadas (columnas 1 a 3 ) con la descrita para la "comunidad de Culcita macrocarpa" del oriente de Asturias (columna 4: columna sintética (excluidos briófitos) a partir de 4 inventarios tomados de Fernández Ordoñez et al. 2003). P: especie presente en el dosel; $P($ dom): especie dominante en el dosel

\section{Caracterización biométrica y reproductiva}

Los resultados relativos a aspectos biométricos y estado vital de los individuos estudiados aparecen sintetizados en la figura 4. A este respecto es necesario advertir que no fue posible tomar datos de cinco individuos de pequeña talla de la subpoblación FG-I, debido a su situación en una pared vertical a varios metros de altura. Por ello, los datos recabados corresponden exclusivamente a 36 de las 41 plantas localizadas, repartidas en 14,21 y 1 respectivamente por subpoblación, habiéndose efectuado las determinaciones sobre un total de 127 frondes.

El tamaño de las poblaciones conocidas de Culcita macrocarpa en la Cornisa Cantábrica es muy variable (Láinz 1968, Fernández Ordoñez et al. 1984, Pérez Carro et al. 1989, Soñora 1992, Quintanilla et al. 2004), aunque no abundan las cuantificaciones numéricas precisas de las mismas. Quintanilla (1997) estima que la mayor población de la Europa continental, integrada por unos 1.000 individuos, se hallaría en el "Parque Natural das Fragas do
Eume" (A Coruña); poblaciones de gran tamaño se encontrarían igualmente en el $\mathrm{E}$ de Asturias (Argüelles et al. 2004-2005). En el extremo opuesto, algunos autores describen poblaciones constituidas por unos pocos individuos (Soñora 1992: "una docena de ejemplares"). A la vista de esta información, las subpoblaciones situadas en la localidad estudiada podrían considerarse como de tamaño medio a pequeño.

La mayor parte de los individuos censados son monocaules (80\%); entre los multicaules, predominan los bicaules $(14 \%)$, sobre los tricaules (3\%) y tetracaules (3\%)(figura 4$)$. La distribución de frondes en clases de longitud (figura 4) muestra la existencia de tendencias diferentes según se trate de frondes estériles (cuyas frecuencias más elevadas se encuentran en las clases de longitud comprendidas entre los 50 y $124 \mathrm{~cm}$ ) o fértiles (con máximos en las clases comprendidas entre 150 y $199 \mathrm{~cm}$ de longitud). Todas las frondes menores de $75 \mathrm{~cm}$ de longitud (17,3\% del total) resultaron estériles mientras que la totalidad de las que superaron los $175 \mathrm{~cm}(19,5 \%$ del total) lo fueron fértiles. La fronde con mayor longitud superó los $300 \mathrm{~cm}$. En cuanto a 
su condición desde el punto de vista reproductivo (figura 4), el $44,5 \%$ de las plantas estudiadas producen frondes que mostraban soros (individuos "adultos") en el momento de realización de los trabajos de campo. Al comparar la longitud de las frondes y su condición adulta/juvenil se observa que las frondes que portan soros (el $48,0 \%$ del total) son siempre de longitud superior a los $75 \mathrm{~cm}$, valor sensiblemente superior al mínimo de $10 \mathrm{~cm}$ establecido por Laínz (1986). No obstante, también existen frondes estériles de gran tamaño, que llegan a superar los $160 \mathrm{~cm}$ de longitud (figura 4). Los conteos de frondes realizados en los individuos de Culcita macrocarpa estudiados muestran que casi el $80 \%$ presentan 3 o más frondes, siendo la subpoblación FG-I la que mayor proporción tiene de individuos con dos frondes (el 28,6 \%). No se han contabilizado individuos solamente con 1 fronde.

En conjunto, los datos obtenidos acerca de aspectos biométricos y reproductivos de la población estudiada son coincidentes con la información disponible para el resto de áreas peninsulares donde está presente este helecho (Quintanilla et al. 2004).

\section{Estado fitosanitario}

Con relación al estado fitosanitario de las plantas estudiadas, en los trabajos de campo realizados se han detectado dos tipos de afecciones sobre las frondes (figura 4). En el primer caso se trata de órganos que han perdido, por causa desconocida, su porción apical ("ápice truncado"). La caída de ramas o fragmentos rocosos de las paredes próximas o la herbivoría practicada por herbívoros silvestres (corzo) o domésticas (caballos, vacas) podrían ser agentes causales de este fenómeno.

Por otra parte, se han observado individuos de Culcita macrocarpa con frondes cuyo limbo se halla desprovisto, total o parcialmente, de la porción fotosintética y se encuentra reducido a los haces vasculares y algunos grupos de esporangios (figura 3). Esta afección está presente en un $11 \%$ de las frondes censadas, prácticamente todas ellas reproductivas (figura 4). Aunque no hemos podido determinar la causa de este fenómeno, pensamos que puede deberse a la acción de un invertebrado, posiblemente un insecto. En todo caso, el hecho de que las frondes afectadas por esta pérdida de superficie fotosintética se hallan totalmente desarrolladas indica que el agente causal habría actuado una vez completado su crecimiento, es decir, a partir de mediados del verano.

En ambos casos, se desconoce el grado de recurrencia de las afecciones comentadas así como la variación interanual que pueda tener su incidencia sobre el total de frondes producido por los individuos. De la misma manera, se ignora en qué medida la pérdida de superficie fotosintética puede estar afectando a la futura viabilidad de las plantas. Ello justifica la realización de un seguimiento a medio/largo plazo para evaluar en qué medida estos procesos pueden condicionar la persistencia de las subpoblaciones estudiadas.

\section{Amenazas}

Dejando a un lado los aspectos fitosanitarios comentados, la persistencia a corto-medio plazo de estos núcleos poblacionales parece estar asegurada mientras se mantengan las condiciones del biotopo que ocupan actualmente. Sin embargo, la cuenca del Río Sor se encuentra sometida desde mediados del pasado siglo a un proceso de sustitución progresiva de la cubierta vegetal preexistente por plantaciones de especies de crecimiento rápido, principalmente Eucalyptus globulus. Precisamente, el reemplazo del arbolado autóctono por plantaciones de especies introducidas es, a juicio de diversos autores (Quintanilla et al. 2004, Amigo 2005, Romero et al. 2005), la principal amenaza para el mantenimiento de las poblaciones del helecho estudiado en el ámbito noroccidental ibérico.

Al efecto negativo que una eventual tala de la masa arbolada en la que se encuentran los núcleos poblacionales estudiados habría que añadir el tránsito del ganado en el caso de que se incrementase la cabaña vacuna y caballar que se cría en semilibertad en la zona, que acostumbra a refugiarse en épocas meteorológicas adversas en los bosques caducifolios, así como la posible incidencia de los incendios forestales (tabla 1), ambos factores con un previsible mayor impacto sobre los ejemplares de mayor tamaño de las subpoblaciones FG-II y FG-III. Aunque esto último pudiera considerarse como poco probable, debido a la baja combustibilidad de la masa arbolada que alberga estas subpoblaciones, su localización en una ladera umbrosa y fuertemente inclinada y su situación en las inmediaciones de un arroyo, las evidencias de incendios pretéritos que todavía en la actualidad se pueden encontrar en el interior de este bosque, impiden descartar los fuegos forestales como factores de amenaza para la conservación de Culcita macrocarpa en la localidad estudiada.

En gran medida, el efecto adverso de factores comentados sobre los núcleos poblacionales estudiados podría verse minimizado si estos se incluyeran dentro de alguna de las figuras legales de protección que contempla la legislación vigente en materia de protección ambiental, ya que, a diferencia de lo que ocurre con el resto de poblaciones de Culcita macrocarpa conocidas en territorio gallego, que se encuentran dentro del perímetro de figuras de protección declaradas con arreglo a la legislación autonómica vigente, como "Parque Natural" o "Zona de Especial Protección de los Valores Naturales", las aquí tratadas no están incluidas dentro de ningún Espacio Natural Protegido. La delimitación de este posible nuevo ENP ("Zona de Especial Protección de los Valores Naturales"?) podría realizarse teniendo en cuenta que en la cuenca del Río Sor existen, además de otros valores naturales, poblaciones de otros helechos incluidos dentro del "Catálogo Galego de Especies Ameazadas", como Dryopteris aemula, Hymenophyllum tunbrigense o Woodwardia radicans, que muestran, al igual que la especie aquí estudiada, una fuerte dependencia de los ambientes nemorales para el desarrollo de sus ciclos vitales (Amigo 2005). 


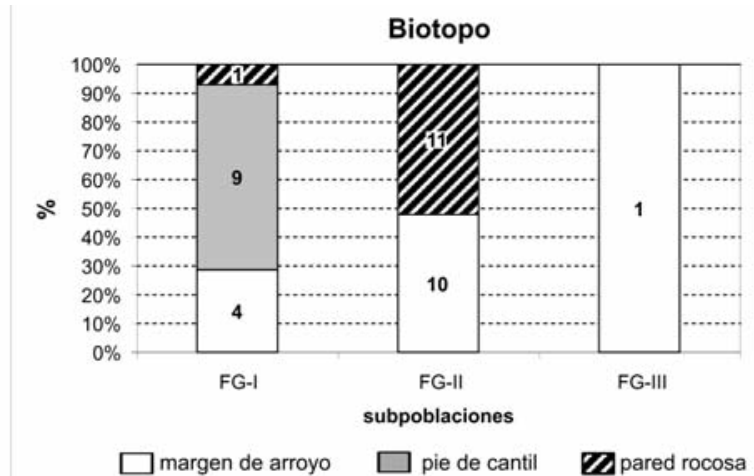

Longitud de las frondes en función de su condición reproductiva

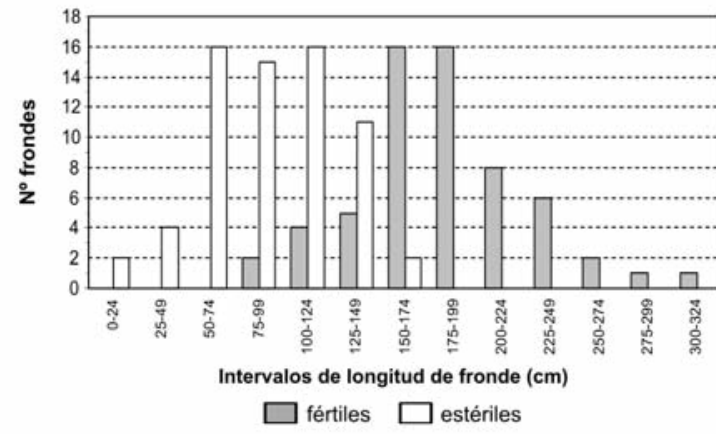

$\mathrm{N}^{\circ}$ de frondes

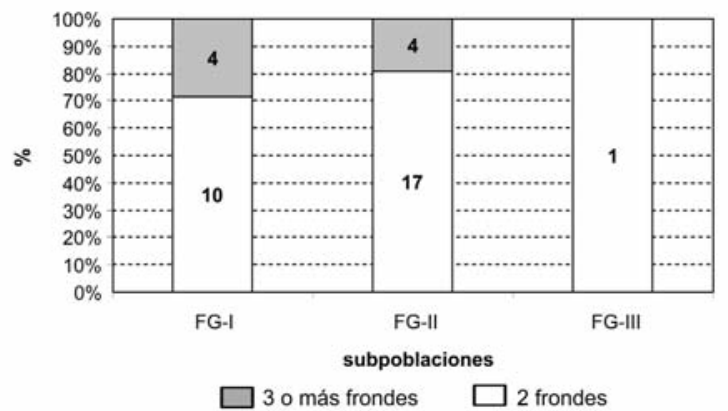

$\mathrm{N}^{\circ}$ de individuos según nivel de ramificación

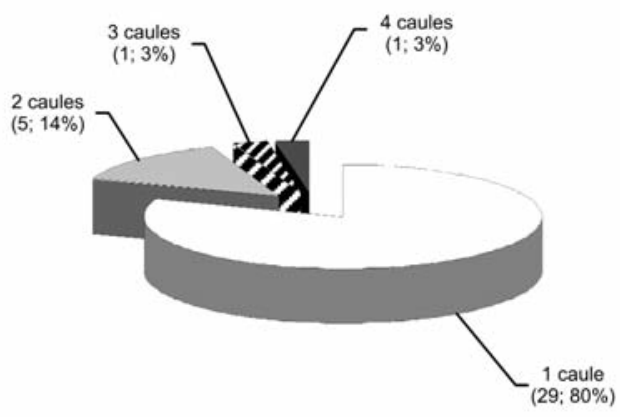

Estado reproductivo

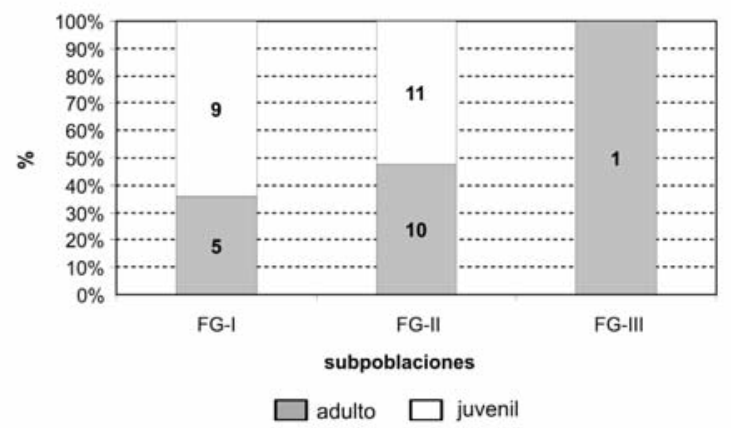

Estado fitosanitario

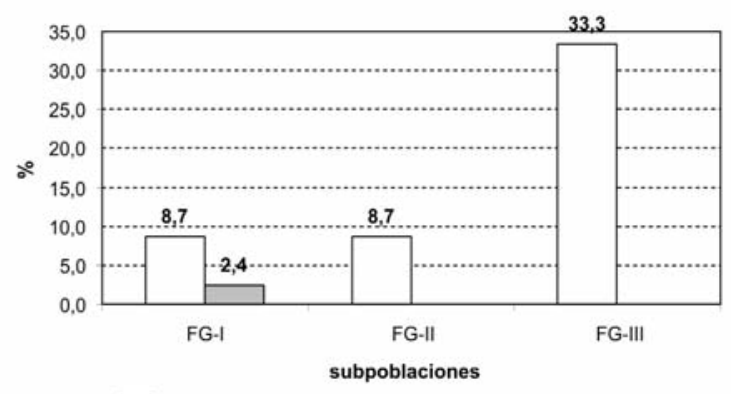

$\square$ pérdida de sup. fotosintética $\square$ ápice truncado

Figura 4.- Representaciones gráficas de la distribución/porcentaje de los individuos de Culcita macrocarpa estudiados en función de diversos parámetros. Arriba izq.: distribución por biotopos; arriba der.: grado de ramificación caulinar; centro izq.: clases de longitud de las frondes en función de su condición reproductiva (estériles, fértiles); centro der.: estado vital de los individuos (juveniles/adultos); abajo izq.: vitalidad ( $\mathrm{n}^{\circ}$ de frondes por individuo); abajo der.: nivel de afección (\%) por defoliación y pérdida de la porción apical

\section{Conclusiones}

La caracterización ambiental de los lugares en los que se encuentran las tres subpoblaciones de Culcita macrocarpa estudiadas muestra que éstas se desarrollan en condiciones topo-edáficas similares a las establecidas para otros enclaves en los que aparece dicha especie en Galicia y otras localidades cantábricas (lugares sombríos y resguardados de difícil acceso protegidos por paredes rocosas o vegetación arbolada densa), a excepción de la cota altitudinal en la que se encuentran, que es la más elevada de las conocidas hasta el momento dentro de este territorio. En cuanto a aspectos demográficos, se trata de un conjunto poblacional no excesivamente numeroso (41 individuos) pero que parece mostrar una tendencia estable por cuanto, además de individuos fértiles de gran tamaño, también contienen una elevada proporción de individuos juveniles (>50\%). En la actualidad, la escasa influencia de la acción antropo-zoógena que existe en el área boscosa en la que se encuentra la población estudiada no supone una amenaza para su mantenimiento. No obstante, la eventual potenciación del uso ganadero o la sustitución de los bosques por plantaciones forestales de especies de crecimiento rápido (principalmente eucaliptos) constituirían 
riesgos importantes de cara a su conservación. Debería estudiarse, no obstante, la causa de las defoliaciones constatadas en un cierto número de frondes por si pudiera tener efectos negativos a medio y largo plazo, así como la declaración de un nuevo espacio natural protegido que incluyera la población aquí estudiada y la aprobación su correspondiente "Plan de Gestión".

\section{Referencias bibliográficas}

Argüelles, J.M., Carlón, L., Gómez Casares, G., González del Valle, J.M., Laínz, M., Moreno Moral, G. \& Sánchez Pedraja, O. (2004-2005): Contribuciones al conocimiento de la flora cantábrica, VII. Boletín de Ciencias Naturales. R.I.D.E.A. n 49: 147-193.

Amigo, J. (2005): Los helechos en el noroeste ibérico y su interés para la conservación. Recursos Rurais. Serie Cursos 2: 11-22.

Amigo, J. \& Norman, G. (1993): La conservación de las Fragas del Río Eume: valoraciones botánicas. Congreso Forestal Español. Ponencias y Comunicaciones, Tomo IV: 15-20. S.E.C.F. Xunta de Galicia. Lourizán.

Amigo, J. \& Norman, G. (1995): Identification of site-types important for rare ferns in an area of deciduous woodland in northwest Spain. Vegetatio 116: 133-146.

Bañares, A., Blanca, G., Güemes, J., Moreno, J.C. \& Ortiz, S. (Eds.)(2004):Atlas y Libro Rojo de la Flora vascular amenazada de España. Dirección General para la Biodiversidad. Ministerio de Medio Ambiente. Madrid. 1069 pp.

Boudrie, M. (1998): Les ptéridophytes du Pays Basque et du nord-ouest de l'Espagne: écologie, répartition, protection. Journal de Botanique 5: 43-52.

Martínez Cortizas, A., Castillo Rodríguez, F. \& Blanco Chao, R. (1999): Precipitación. En: A. Pérez Alberti \& A. Martínez Cortizas: Atlas Climático de Galicia: 75-91. Xunta de Galicia. Santiago de Compostela.

Díaz González, T.E. \& Fernández Prieto J.A. (1994): La vegetación de Asturias. Itinera Geobot. 8: 243-528.

Fernández Ordóñez, M.C., Fernández Prieto, J.A. \& Collado, M.A. (1984): Una nueva localidad de Culcita macrocarpa en Asturias: datos sobre su ambiente vegetal. Boletín de Ciencias Naturales. I.D.E.A. 33: 49-61.

Fernández Ordoñez, M.C., Díaz González, T.E. \& Collado Prieto, M.A. (2003): Evaluación de la biodiversidad briopteridofítica de la cuenca del Río de Nueva (Llanes, Asturias, N Península Ibérica): bases para su protección u conservación. Acta Botanica Barcinonensia 49: 191-208

I.G.M.E. (1976): Mapa Geológico de España. E. 1:50.000. Hoja 8(7-3). Vivero. Servicio de Publicaciones. Ministerio de Industria. Madrid.

Laínz, M. (1968): Aportaciones al conocimiento de la Flora Gallega, VI: 1-39. I.F.I.E. Madrid.
Laínz, M. (1986): XVII. Culcitaceae. En: S. Castroviejo, M. Laínz, G. López-González, P. Montserrat, F. Muñoz Garmendia, J. Paiva \& L. Villar: Flora Iberica. Vol. I: 79-80. Real Jardín Botánico de Madrid. C.S.I.C. Madrid.

Pérez Carro, J., Díaz González, T.E. \& Fernández Areces, M.P. (1989): Acerca de Equisetum x font-queri Roth., más precisiones corológicas sobre Culcita macrocarpa C. Presl. Anales del Jardín Botánico de Madrid 45(2): 550-551.

Prelli, R. (2002): Les Fougères et plantes alliés de France et d'Europe occidentale. Editions Belin. 432 pp. Paris.

Quintanilla, L.G. (1997): Distribución de los helechos relictos macaronésicos en el Parque Natural Fragas do Eume (A Coruña): Importancia biogeográfica en la pteridoflora gallega. Tesis de licenciatura inédita. 104 pp. Departamento de Bioloxía Vexetal. Universidade de Santiago de Compostela.

Quintanilla, L.G. \& Amigo, J. (2001): Culcita macrocarpa C. Presl (Dicksoniaceae, Pteridophyta) en la cuenca del Xubia (NO de la Península Ibérica). Nova Acta Científica Compostelana (Biol.) 11: 267-268.

Quintanilla, L.G., Amigo, J., Pangua, E. \& Pajarón, S. (2002): Análisis biogeográfico de la pteridoflora de la sierra de la Capelada (La Coruña, España). Lazaroa 23: 17-24.

Quintanilla, L.G., Cabezudo, B., García, A., Mesa, R., Nava, H.S. \& Navas, P. (2004): Culcita macrocarpa C. Presl. En: A. Bañares, G. Blanca, J. Güemes, J.C. Moreno \& S. Ortiz (Eds.): Atlas y Libro Rojo de la Flora Vascular Amenazada de España: 662-663. 2a Ed. Dirección General para la Biodiversidad. MMA. 1069 pp.

Rodríguez Guitián, M.A. (2004): Aplicación de criterios botánicos para a proposta de modelos de xestión sustentable das masas arborizadas autóctonas do Subsector Galaico-Asturiano Septentrional. Tesis doctoral inédita. 620 pp. Escola Politécnica Superior de Lugo. USC. Lugo.

Rodríguez Guitián, M.A. (2005): Avaliación da diversidade sílvica do subsector galaico-asturiano septentrional: tipos de bosques, valor para a conservación e principais ameazas. Recursos Rurais. Serie cursos e monografias do IBADER, 2: 23-44.

Rodríguez Guitián, M.A. (2010a): Aportacións sobre a tipoloxía e composición florística dos bosques mesófilos de quercíneas do occidente da Cornixa Cantábrica (NW Ibérico). Recursos Rurais 2(6): 35-73.

Rodríguez Guitián, M.A. (2010b): Temperate riverside forests without alder trees at the NW of the Iberian peninsula: ecology, phytosociological profile and interest for preservation policies. Lazaroa 30: en prensa.

Rodríguez Guitián, M.A. \& Ramil Rego. P. (2007): Revisión de las clasificaciones climáticas aplicadas al territorio gallego desde una perspectiva biogeográfica. Recursos Rurais 1(3): 31-53.

Romero, M.I., Rodríguez Guitián, M.A. \& Rubinos, M. (2004): Adiciones al catálogo pteridológico gallego. Bot. Comp. 28: 51-55. 
Romero, M.I., Amigo, J., Rodríguez Guitián, M.A., Díaz Varela, R., Ferreiro da Costa, J. (2005): Conservación de la pteridoflora amenazada en el NW Ibérico (Galicia): las especies incluídas en la Directiva Hábitats. Bull. Soc. Hist. Nat. Toulouse 141-2: 227-232.

Soñora, F.X. (1992): Notas pteridológicas de Galicia. IV. Acta Bot. Mal. 17: 282-283.
Viane, R., Mayor López, M. \& Jermy, C. (1987): Excursion Guide. XIV International Botanical Congress. Excursion $n^{\circ}$ 39. Berlin. 54 pp.

Xunta (2007): Decreto 88/2007 do 19 de abril, polo que se regula o Catálogo galego de especies ameazadas. DOGA $n^{\circ}$ 89: 7.409-7.423. Consellería de Medio Ambiente e Desenvolvemento Sostible. Santiago de Compostela.

\section{ANEXO}

Información biométrica recogida en los individuos de las subpoblaciones de Culcita macrocarpa (L.) Presl.estudiadas. Sit. top. (situación topográfica): C: cauce de arroyo; P: pie de cantil; R: pared rocosa. Observaciones: 々fronde con el ápice truncado; :): fronde afectada por pérdida de superficie fotosintética. Estado vital: J: juvenil, individuo sin soros; A: adulto, individuo con soros

\begin{tabular}{|c|c|c|c|c|c|c|c|c|c|}
\hline Subpoblación & $N^{\circ}$ ind & Sit. top. & $\mathrm{N}^{\circ}$ de caules & Caule & Estado vital $\mathrm{N}$ & $\mathrm{N}^{\circ}$ de fronde & Long. (cm.) & Soros Vitalidad & Observaciones \\
\hline FG-I & 1 & $C$ & 1 & 1 & $\mathrm{~J}$ & 1 & 55 & No alta & - \\
\hline & & & & & & 2 & 50 & No & - \\
\hline & & & & & & 3 & 85 & No & - \\
\hline & 2 & $C$ & 1 & 1 & $J$ & 1 & 70 & media & - \\
\hline & & & & & & 2 & 75 & No & - \\
\hline & & & & & & 3 & 95 & No & - \\
\hline & 3 & $C$ & 2 & 1 & $\mathrm{~J}$ & 1 & 131 & media & - \\
\hline & & & & & & 2 & 100 & No & - \\
\hline & & & & 2 & $\mathrm{~J}$ & 1 & 140 & media & - \\
\hline & & & & & & 2 & 115 & No & - \\
\hline & 4 & $C$ & 1 & 1 & $\mathrm{~J}$ & 1 & 128 & media & - \\
\hline & & & & & & 2 & 115 & No & - \\
\hline & 5 & $P$ & 1 & 1 & $\mathrm{~J}$ & 1 & 140 & alta & - \\
\hline & & & & & & 2 & 115 & No & - \\
\hline & & & & & & 3 & 65 & No & 々々 \\
\hline & 6 & $P$ & 1 & 1 & $\mathrm{~J}$ & 1 & 48 & media & - \\
\hline & & & & & & 2 & 120 & No & - \\
\hline & 7 & $P$ & 3 & 1 & A & 1 & 195 & alta & - \\
\hline & & & & & & 2 & 169 & $\mathrm{Si}$ & - \\
\hline & & & & & & 3 & 192 & $\mathrm{Si}$ & . \\
\hline & & & & 2 & $\bar{A}$ & 1 & 187 & alta & - \\
\hline & & & & & & 2 & 192 & $\mathrm{Si}$ & - \\
\hline & & & & & & 3 & 75 & $\mathrm{Si}$ & 々々 \\
\hline & & & & 3 & $\bar{A}$ & 1 & 190 & alta & - \\
\hline & & & & & & 2 & 176 & $\mathrm{Si}$ & - \\
\hline & & & & & & 3 & 95 & $\mathrm{Si}$ & 々々 \\
\hline & 8 & $P$ & 1 & 1 & $\mathrm{~J}$ & 1 & 54 & media & - \\
\hline & & & & & & 2 & 48 & No & - \\
\hline & 9 & $P$ & 1 & 1 & $A$ & 1 & 217 & alta & - \\
\hline & & & & & & 2 & 242 & $\mathrm{Si}$ & - \\
\hline & & & & & & 3 & 226 & $\mathrm{Si}$ & - \\
\hline & 10 & $R$ & 1 & 1 & $\mathrm{~J}$ & 1 & 75 & alta & - \\
\hline & & & & & & 2 & 95 & No & - \\
\hline & & & & & & 3 & 100 & No & - \\
\hline & 11 & $P$ & 1 & 1 & A & 1 & 197 & alta & (:): \\
\hline & & & & & & 2 & 170 & $\mathrm{Si}$ & - \\
\hline & & & & & & 3 & 216 & $\mathrm{Si}$ & - \\
\hline & 12 & $P$ & 1 & 1 & $A$ & 1 & 162 & alta & - \\
\hline & & & & & & 2 & 167 & $\mathrm{Si}$ & $: ;:)$ \\
\hline & & & & & & 3 & 166 & $\mathrm{Si}$ & 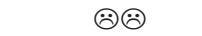 \\
\hline & 13 & $P$ & 1 & 1 & $A$ & 1 & 215 & alta & - \\
\hline & & & & & & 2 & 210 & $\mathrm{Si}$ & - \\
\hline & & & & & & 3 & 197 & $\mathrm{Si}$ & - \\
\hline & 14 & $P$ & 1 & 1 & $\mathrm{~J}$ & 1 & 98 & alta & - \\
\hline & & & & & & 2 & 65 & No & - \\
\hline & & & & & & 3 & 67 & No & $: ;:)$ \\
\hline
\end{tabular}


Subpoblación $\mathrm{N}^{\circ}$ ind. Sit. top. $\mathrm{N}^{\circ}$ de caules Caule Estado vital $\mathrm{N}^{\circ}$ de fronde Long. $(\mathrm{cm}$.) Soros Vitalidad Observaciones FG-II

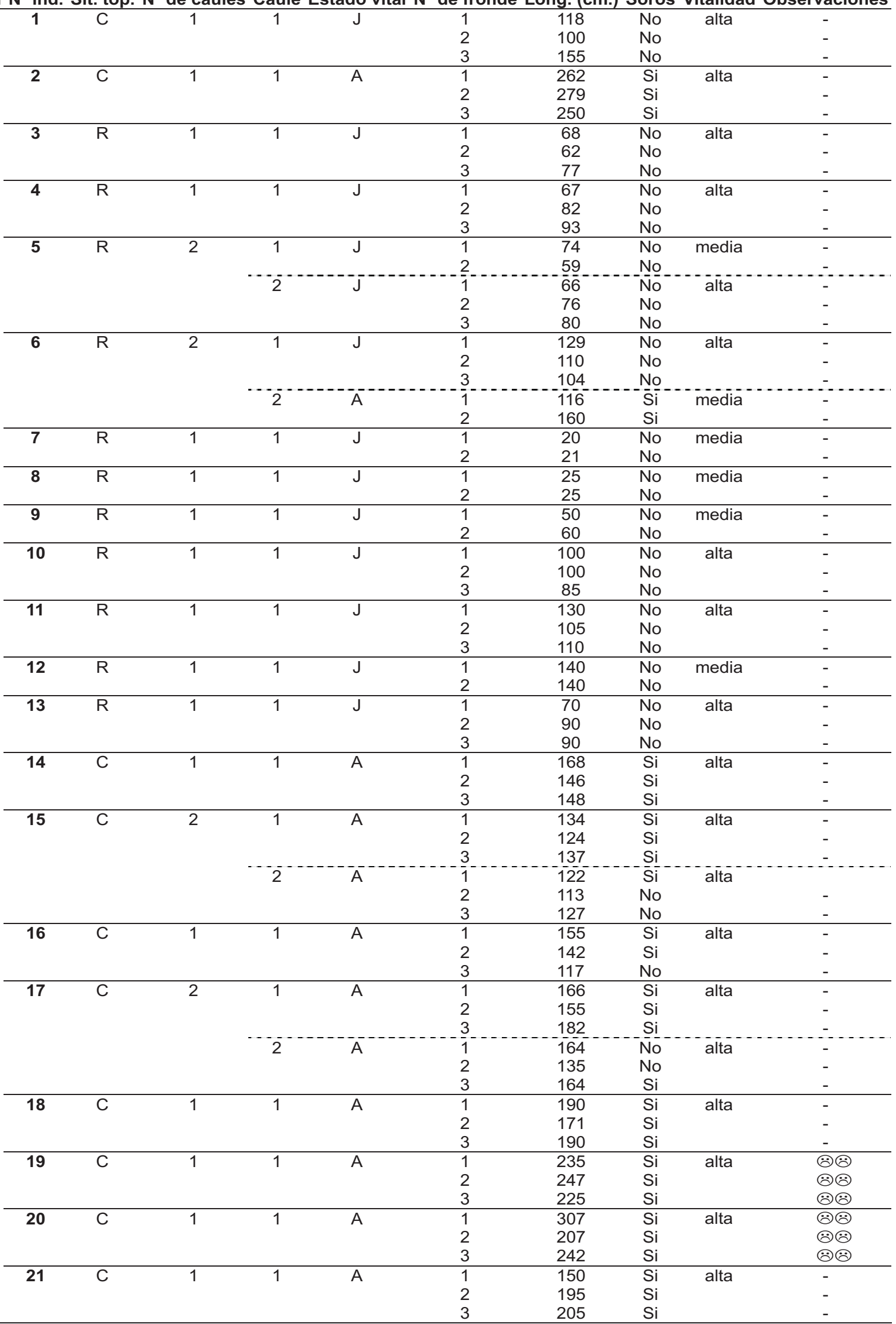


Subpoblación $\mathbf{N}^{\circ}$ ind. Sit. top. $\mathbf{N}^{\circ}$ de caules Caule Estado vital $\mathbf{N}^{\circ}$ de fronde Long. (cm.) Soros Vitalidad Observaciones FG-III

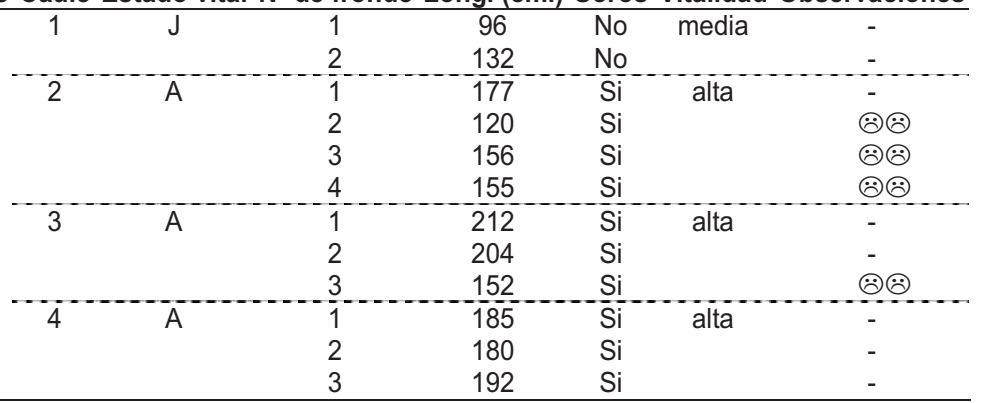

